\title{
Distillation Column Malfunctions Identification using Higher Order Statistics
}

\author{
M. E. Hammad, H. Kasban, Sayed M. \\ Elaraby \\ Engineering Department, Nuclear Research center, \\ Atomic Energy Authority \\ Cairo, Egypt
}

\author{
Moawad I. Dessouky, O. Zahran, Fathi E. \\ Abd El-Samie \\ Department of Electronics and Electrical \\ Communications, Faculty of Electronic Engineering, \\ Menoufia University \\ Menouf, Egypt
}

\begin{abstract}
This paper presents a proposed approach for distillation column malfunction identification using Higher Order Statistics (HOS). Gamma ray scanning techniques have been used for examining the inner details of a distillation column. In the proposed method, the signals are firstly divided into frames; each frame contains only the signal of one column tray. Secondly, HOS are estimated for these frame signals. Then features are extracted from the HOS estimate. Finally, features are used for training and testing of Artificial Neural Network (ANN) to identify the distillation column malfunctions. The simulation results show that the HOS can be used efficiently for the distillation column malfunction identification especially at high noisy scanning conditions.
\end{abstract}

\section{Keywords}

Bispectrum, Cumulant, moment, and Trispectrum.

\section{INTRODUCTION}

Distillation columns are considered as one of the most critical components in petroleum refineries, gas processing installations and chemical plants. Plant performance depends to a large extent on the ability of these columns to function as intended. Defective columns may lead to serious consequences to the plant operation and hence the quality of the product. Thus, when a distillation column experiences irregularities, it is urgently necessary to find out exactly what is happening inside the column. The earlier the problem is identified and corrected, the lesser the loss and the cost to rectify it. There are several techniques used for distillation column scanning such as x-ray imaging, tomography and gamma scanning (GS) technique. This paper concerning with gamma scanning technique according to its distinguishing features compared to other conventional techniques such as it can be carried out on-line without disruption to the process, can be carried out on the entire column length or portions of the column, no preparation required, non-contact measurements are performed, column scanning remains at all times under the control of the operator and it is a nondisruptive process and non-invasive technique $[8,9]$.

In GS technique, distillation column scanning is carried out using a small suitable sealed gamma radio isotope and a radiation detector. Both the source and the detector are moved concurrently in small increments on opposite sides, along the exterior length of the scanned unit and a relative density profile of the contents of the column is obtained. The obtained profile (signals) demonstrate the inner details of a distillation column (i.e. inside view of the distillation column) where the areas containing relatively high density materials, such as liquid and/or metal, provide relatively low radiation intensities, while the areas with relatively low densities, such as vapor spaces between trays, result in high radiation intensity levels. In the case of presence of any type of malfunction in the distillation column, the normal behavior profile will change according to the type of malfunction.

This paper presents a proposed approach for the automatic identification of distillation column malfunctions. This topic is rarely interested by researchers. Although the distillation column malfunction using gamma rays is presented by many researchers before; in 2005, Pablo et al. use noninvasive gamma ray technique to measure the liquid distribution in a packed column using three different radioactive sources (60Co, 131I, and 99mTc). The best results were obtained using the 99mTc source [1]. In 2008, Salahshoor et al. develop an online multivariable identification approach for any dynamic changes based on an adaptive Takagi-Sugeno fuzzy model. This identification approach has been applied on a nonlinear distillation column to demonstrate its effectiveness [2]. In 2011, Muddu et al. develop a multi-rate adaptive predictive control model. The simulation results give satisfactorily performance when the operation of the distillation column is shifted from moderate purity region to high purity region [3]. In 2012, Ugryumova et al. present mathematical models that predict the behavior of the column to improve the working conditions of distillation columns [4]. Recently, column scanning by gamma rays has become a well known technique and has been widely applied in industrial routines for these purposes [5-7].

This paper is organized as follow; Section 2 will introduce the principles gamma ray scanning of a distillation column and its procedure with its distinguishing features compared to other conventional techniques. Section 3 presents the approach for identifying the distillation column malfunctions. Section 4 presents the Experimental Work. Section 5 presents the results and discussions. Finally, section 6 presents the conclusion remarks.

\section{DISTILLATION COLUMN SCANNING USING GAMMA RAYS}

When scanning a distillation column or similar vessel using gamma rays, a suitably gamma ray emitting radioactive sealed source in a panoramic source container and a scintillation detector are lowered down concurrently in small increments on opposite sides along the length of the column [8]. The quantity of the absorbed or transmitted gamma rays is an indication of the actual quantity and nature of the material between the radioactive source and the detector. The relation, which describes the transmission of gamma rays through a material, is expressed as [9]:- 


$$
I=\mathbf{I}_{0} e^{(-\mu V x)}
$$

Where, I is the intensity of radiation transmitted through the material between the radioactive sealed source and scintillation detector, I0 is the intensity of the incident radiation without a medium to absorb radiation between the radioactive sealed source and scintillation detector (constant), $\mu$ is the absorption coefficient of the material, $\mathrm{V}$ is the density of the absorbing material between the source and scintillation detector (variable), and $\mathrm{x}$ is the radiation path length through which the transmitted radiation travel. Equation (1) states that the radiation intensity measured by a detector is inversely proportional to the material density of the absorber (i.e. the medium that exists between the source and the detector). During the scanning execution, the transmitted radiation intensity from the radioactive sealed source received by the scintillation detector are graphically recorded on a laptop computer connected to the detector through a data acquisition system as shown in Fig. 1.

The intensity of the gamma rays received at the detector location varies depending upon the variation of the material density between the source and the detector. This intensity variation can be analyzed for the detection of the distillation column malfunctions.

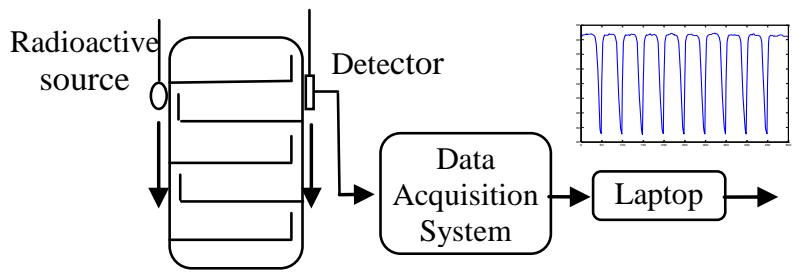

Fig 1: Principles of gamma ray scanning of distillation column

In this paper, the proposed approaches for distillation column malfunction identification are applied on both real signals and simulated signals generated using software approved by International Atomic Energy Authority (IAEA) through the AFRA projects $\mathrm{RAF} / 8 / 040$ and $\mathrm{RAF} / 1 / 004$. This paper considered all common malfunctions which are; foaming, weeping, column tray damage, column tray collapse, and flooding. The signals obtained by gamma scanning of distillation column consists of group of minima points as illustrated in Fig. 2. These minima points represent the position of trays inside the distillation column which carry liquid over it, so above trays the intensity of radiation decrease with slop can be used to represent the liquid level on its tray. Between trays there are regions of vapour which can be found on the signal obtained by regions of high radiation intensity. The normal behavior and the malfunctions of the distillation column are discussed with some details below:

\subsection{Normal Column Behavior}

Typical scan of a normal operating column without any processing problems is shown in Fig. 2. The orientation of scan-line is perfect where the scanning is executed across the life area of the trays, missing down comers. This can be confirmed by the fact that, above and below the trays, the radiation intensity returns to approximately the same high level (Low density vapour baseline). No serious damage or malfunction was observed.

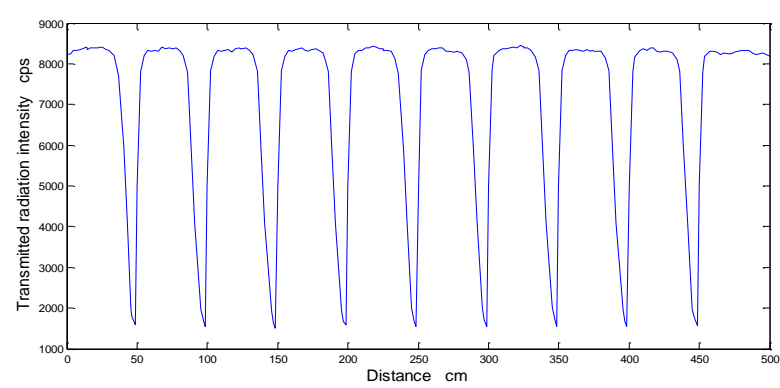

Fig 2: Signal of normally operated distillation columns

\subsection{Distillation Column Foaming}

Foaming refers to the expansion of liquid due to passage of vapour or gas separation is reduced. This can be observed above the tray number 6 as indicated in Fig. 3, where the radiation intensity above the tray are not the same as expected and the gradient increase is above the tray towards the vapour line.

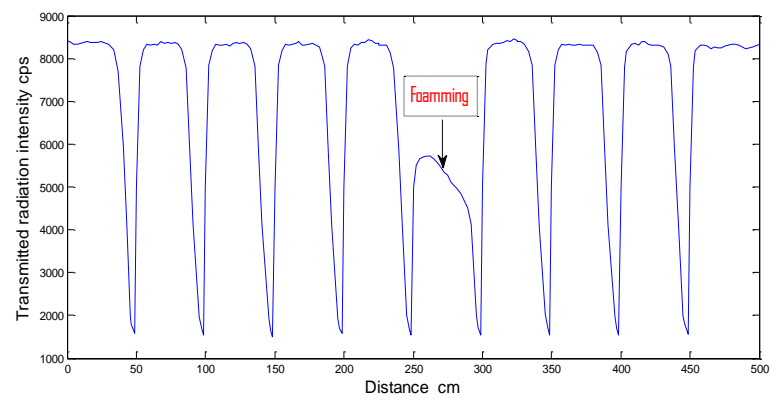

Fig 3: Distillation column foaming malfunction

\subsection{Distillation Column Weeping}

Weeping is strictly a mirror image of foaming where the radiation intensity slope is measured below the tray as indicated in Fig. 4. Weeping can be caused by higher liquid flow rate, higher load of liquid on tray, insufficient liquid/vapour sealing and ineffective working of contactors (i.e. valves and bubble caps). All this reasons makes the pressure exerted by vapour is insufficient to hold liquid on a tray. Weeping is a distillation column phenomena that is not easy detectable by scanning and needs skill and experience.

\subsection{Distillation Column Damage}

Tray damage can be defined as a partially damaged tray. One of the main reasons of tray damage is the susceptible tray vibration which can be attributed to missing valves on valve tray, missing tray panels or man ways, large holes in trays from corrosion, heavily fouled (dirty) trays due to deposits, debris and/or coking, and long term fatigue fosters failures. Damage can be observed in tray number 8 as indicated in Fig. 5. 


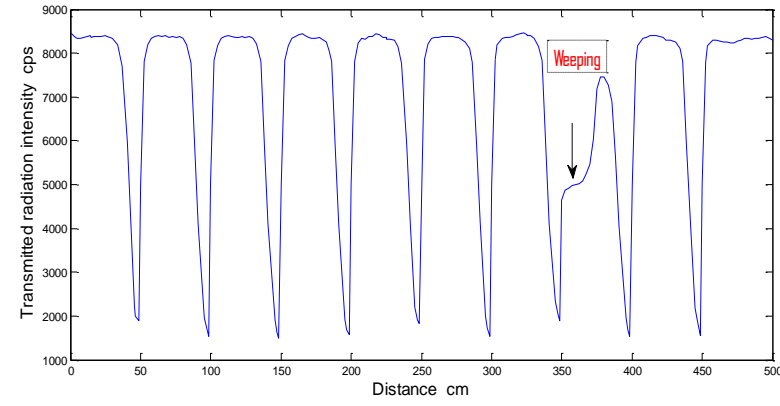

Fig 4: Distillation column weeping malfunction

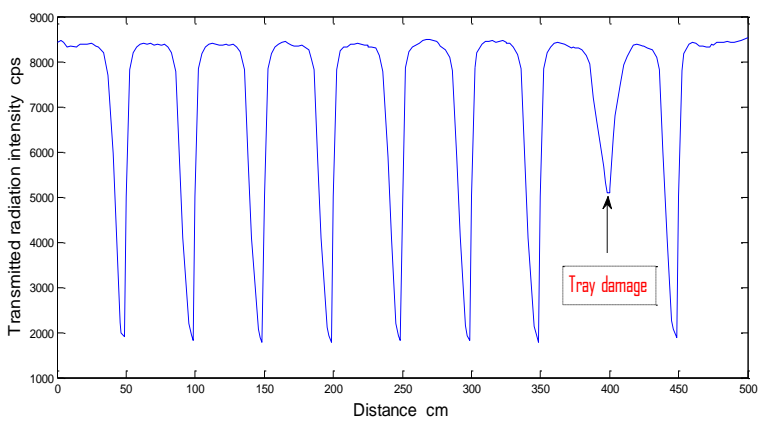

Fig 5: Distillation column damage malfunction

\subsection{Distillation Column Collapse Malfunction}

Tray collapse can be defined as fully damage tray as shown in Fig. 6, where a high transmitted radiation intensity is recorded in the position of the eighth column tray. This can be attributed that no absorption material was present in this area to absorb the transmitted radiation; this is indicative that the tray has collapsed.

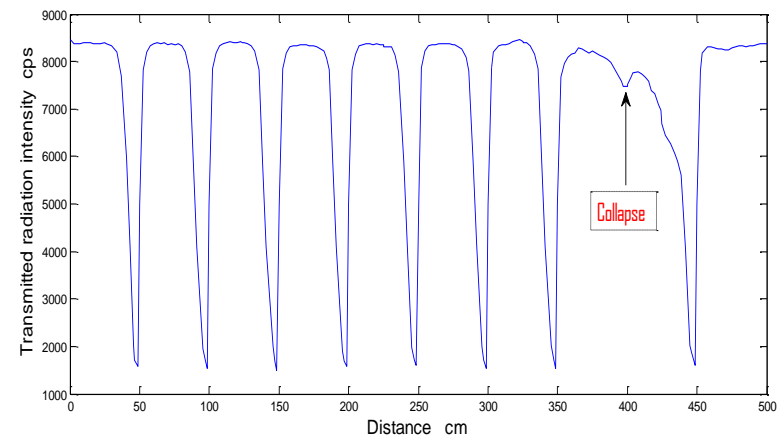

Fig 6: Distillation column collapse malfunction

\subsection{Distillation Column Flooding Malfunction}

Tray flooding can be described as the excessive amount of liquid on a tray. Tray flooding undermines good liquid/vapour disengagement between trays and can be the reason for distillation products to be off spects.

Figure 7 illustrate that a tray is damaged severely and that is the cause of flooding due to a blockage on tray 8 or in its down comer. Flooding is also indicative of poor liquid/vapour disengagement and indication of a relatively high pressure difference over the column.

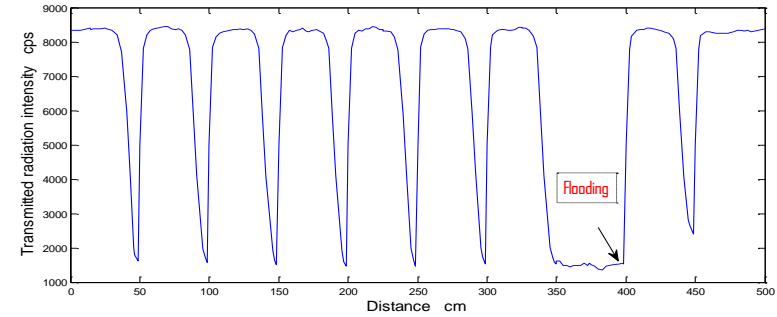

Fig 7: Distillation column flooding malfunction

\section{THE PROPOSED DISTILLATION COLUMN MALFUNCTION IDENTIFICATION APPROACH}

The proposed method for the Distillation column malfunction identification based on higher order statistics has two phases; a training phase and a testing phase. In the training phase, a database of distillation column signals is built with features extracted from the bispectrum and trispectrum estimates of these signals. In the testing phase, the features are extracted from every incoming corrupted signal, and the feature matching step is performed to decide whether these features belong to a normal column or to one of distillation column malfunction, in addition to estimate the type of this malfunction. This approach is summarized in Fig 8.

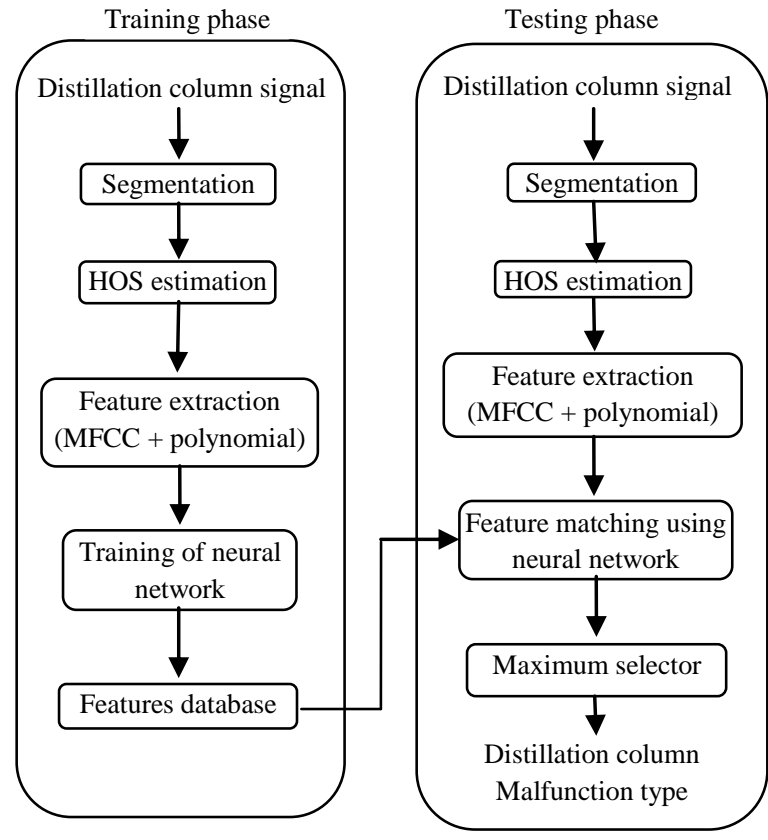

Fig 8: Schematic diagram of the proposed approach for distillation column malfunction identification

\subsection{Data Segmentation}

The maintenance of a distillation column is so difficult process because of its huge height, so in this approach not only we want to know if the distillation column has a malfunction or no, but also we strongly in a need to know the position of that malfunction to make the repairmen of the distillation column so easy for the stuff of maintenance and for the production line. By determining the position of the problem we can save time of maintenance, effort, and money. This means that the malfunction tray should be known. In order to achieve this, the distillation column signal which obtained by gamma ray scanning is firstly segmented into frames. Each frame contains only a signal of one tray of the column. 
As indicated in Fig.2 the density profile consists of a group of minima, each minimum represents the existence of a tray. By determining the position of each minimum, the positions of trays have been determined. The period of each tray is determined by assigning the mid points between each two successive trays.

\subsection{Higher Order Statistics}

HOS are extensions of second order measures (such as the autocorrelation function and the power spectrum) to higher orders, and it defined for stationary random signal in terms of cumulants and moments. Bispectrum and trispectrum are particular cases of higher order spectra [10]. In this paper, we choose higher order statistics for the following reasons:

1. To suppress additive colored Gaussian noise of unknown power spectrum

2. To identify non-minimum phase systems that means reconstruct non-minimum phase signals.

3. To extract information due to deviation from gaussianity.

4. To detect and characterize nonlinear properties in signals as well as identify nonlinear systems.

This section introduces the definition and computation of higher order statistics (i.e. computation of moment and cumulant) and their corresponding higher order spectra for stationary random signals. If $\{\mathrm{x}(\mathrm{k}), \mathrm{k}=\ldots .,-2,-1,0,1$, $2, \ldots \ldots \ldots \ldots\}$ is real stationary discrete time signal and its moment up to order $\mathrm{n}$ exist, then:

$$
m_{n}^{x}\left(\tau_{1}, \tau_{2}, \ldots, \tau_{n-1}\right)=E\left(x(k) x\left(k+\tau_{1}\right), \ldots, x(k) x\left(k+\tau_{n-1}\right)\right)
$$

Represents the nth order moment function of the stationary signal, which depends only on the time differences $\tau 1, \tau 2 \ldots$ $\tau \mathrm{n}-1, \tau \mathrm{i}=0, \pm 1, \pm 2 \ldots$ for all $\mathrm{i}$. Clearly the 2 nd order moment function, $\mathrm{m}_{2}^{\mathrm{x}}(\tau 1)$, is the autocorrelation of $\{\mathrm{x}(\mathrm{k})\}$ Whereas $\mathrm{m}_{3}^{\mathrm{x}}(\tau 1, \tau 2)$ and $\mathrm{m}_{4}^{\mathrm{x}}(\tau 1, \tau 2, \tau 3)$ are the 3 rd and 4 th order moments, respectively. The general relationship between the moments and the cumulants is given by [11];

$C_{n}^{x}\left(\tau_{1}, \tau_{2}, \ldots, \tau_{n-1}\right)=m_{n}^{x}\left(\tau_{1}, \tau_{2}, \ldots, \tau_{n-1}\right)-m_{n}^{G}\left(\tau_{1}, \tau_{2}, \ldots, \tau_{n-1}\right)$

Where $m_{n}^{x}\left(\tau_{1}, \tau_{2}, \ldots, \tau_{n-1}\right)$ is the $n$th order moment function of $\mathrm{x}(\mathrm{k})$ and $\mathrm{m}_{\mathrm{n}}^{\mathrm{G}}\left(\tau_{1}, \tau_{2}, \ldots, \tau_{\mathrm{n}-1}\right)$ is nth order moment function of an equivalent Gaussian signal that has the same mean value and autocorrelation function as $\mathrm{x}(\mathrm{k})$ process. Moments and cumulants are symmetric functions in their arguments and the following three key properties distinguish cumulants from moments [11];

1. If $\{\mathrm{x}(\mathrm{k}), \mathrm{x}(\mathrm{k}+\tau 1), \ldots \ldots, \mathrm{x}(\mathrm{k}+\tau \mathrm{n}-1)\}$ can be divided into any two or more groups that are statistically independent, their nth order cumulants $c_{n}^{x}(\tau 1, \tau 2, \ldots ., \tau \mathrm{n}-1)=0$, whereas $m_{n}^{G}\left(\tau_{1}, \tau_{2}, \ldots, \tau_{n-1}\right) \neq 0$

2. If $\mathrm{x}(\mathrm{k})$ and $\mathrm{y}(\mathrm{k})$ are independent stationary random processes and $\mathrm{z}(\mathrm{k})=\mathrm{x}(\mathrm{k})+\mathrm{y}(\mathrm{k})$, then

$$
c_{n}^{z}\left(\tau_{1}, \tau_{2}, \ldots, \tau_{n-1}\right)=c_{n}^{x}\left(\tau_{1}, \tau_{2}, \ldots, \tau_{n-1}\right)+c_{n}^{y}\left(\tau_{1}, \tau_{2}, \ldots, \tau_{n-1}\right)
$$

3. If the set of random variables $\left\{x(k), x\left(k+\tau_{1}\right), \ldots ., x(k+\right.$ $\left.\left.\tau_{\mathrm{n}-1}\right)\right\}$ is jointly Gaussian, all its joint cumulants of order greater than the second are identically zero.

Higher order spectra (cumulant spectra) of random signals are usually defined in terms of cumulants not moments according to the above proprieties of cumulants that moment does not share. Assuming that the cumulant sequence, $c_{n}^{\mathrm{x}}\left(\tau_{1}, \tau_{2}, \ldots, \tau_{\mathrm{n}-1}\right)$ is absolutely summable, then the nth order cumulant spectra $c_{n}^{\mathrm{x}}\left(\omega_{1}, \omega_{2}, \ldots, \omega_{\mathrm{n}-1}\right)$, of $\mathrm{x}(\mathrm{k})$ exists and its defined as $(\mathrm{n}-1)$ dimensional Fourier transform of the nth order cumulant sequence. The power spectrum, bispectrum and trispectrum are special cases of the nth order cumulan spectrum [10].

There are two main approaches that can be used to estimate higher order spectra of a process when a finite set of measurements is given: the conventional nonparametric method (Fourier type), and parametric approach which is based on Autoregressive (AR), Moving Average (MA) and Autoregressive Moving Average (ARMA) models [11].

\subsubsection{Conventional Non-Parametric Estimators for Higher Order Spectra:}

The conventional non-parametric methods may be classified into two classes, Indirect and Direct methods which may be seen as direct approximation of the higher order spectra definition. While these methods are straightforward, limitation on statistical variance of the estimates, computer time, and memory impose severe problems in their implementation. In fact, the computation may be surprisingly expensive despite the use of fast Fourier transform (FFT) algorithms.

The indirect method can be summarized in segmenting the data into records, subtracting the average value of each record from the data, estimating the higher order moments of each record by using Equation (2), averaging the obtained moments overall segments, estimate $n$th order cumulant $\mathrm{c}_{\mathrm{n}}^{\mathrm{x}}(\tau 1, \tau 2, \ldots$, $\tau \mathrm{n}-1)$ from the estimated moment, then generate the higher order spectrum estimate.

The direct method which is the other class of conventional estimators is found to be very useful for the generation of moment spectra using FFT algorithms. In this approach we use both types of conventional non parametric methods (i.e. direct and indirect methods).

\subsubsection{Parametric Methods for Higher Order Spectrum Estimation:}

One of the widely used approaches in system identification and power spectrum estimation has been the construction of a white-noise-driven, linear time-invariant model from a given realization of a random signal. However, this approach was based primarily on a Gaussian assumption about the data and thus autocorrelation modeling. The main limitation associated with this assumption is that non-minimum phase systems will be identified as being minimum phase when the input to the system is inaccessible. Consequently, the main motivation behind the use of non-Gaussian, white noise-driven parametric models for higher order spectrum estimation is to recover not only the magnitude but also the phase response of the system accurately. Estimating the parameters of AutoRegressive (AR), Moving Average (MA) and AutoRegressive Moving Average (ARMA) models from the cumulant statistics of the noisy observations of the system output is considered and studied in details in [10-11]. The non-minimum phase system identification problem in the bispectrum domain using MA, AR, ARMA models is given in [11]. In this approach we use only ARMA modeling from the parametric methods.

\subsection{Feature Extraction}

Feature extraction means reducing the amount of data presented in the distillation column signal, while retaining the signal discriminative information. In this paper, the feature extraction process is performed using Mel-Frequency Cepstral Coefficients (MFCCs) because they are common and more suitable for these data types. But MFCCs are sensitive to mismatches and time shifts between training and testing data. 
Thus, there is a need for other coefficients to be added to the MFCCs to reduce this sensitivity. Polynomial coefficients have been used for this purpose as these coefficients can help in increasing the similarity between the training and the testing signals.

The Mel-scale is a mapping between the real frequency scale in $\mathrm{Hz}$ and the perceived frequency scale in Mels. The Mapping is virtually linear below $1 \mathrm{kHz}$ and logarithmic above as given by the following equation [11], [12];

$$
f_{\text {Mel }}=2.595 \log \left(1+\frac{f_{\text {linear }}}{700}\right)
$$

The MFCCs are commonly extracted from signal through cepstral analysis. Calculation of the MFCCs proceeds similar to the cepstral transformation process. For the calculation of the MFCCs, the signal is first windowed, the discrete Fourier transform (DFT) is then applied, and the magnitude of the resultant spectrum is estimated on the Mel-scale using a Melfilter bank. The $\log$ of this spectrum is then taken, and the DCT is applied. If each MFCC is modeled as a time waveform over adjacent frames, polynomial coefficients are used to model the slope and curvature of this time waveform for each MFCC. Adding these polynomial coefficients to the MFCCs vector will be helpful in reducing the sensitivity to any mismatch between the training and testing signals.

\subsection{Feature Matching using Artificial Neural Networks}

In the proposed system, the identification step is in fact a features matching process. Multi-Layer Perceptrons (MLPs) neural network is used for feature matching because it is suitable for the problem considered in this paper, this MLPs consists of an input layer, a single hidden layer and an output layer.

In this approach the input vector $X$ is a vector of the discriminating features for each distillation column signal, and the output vector is a vector containing an output for normal operation of distillation column and other outputs for each type of distillation column malfunctions, so the output vector in this approach consists of six outputs.

Training a neural network is accomplished by adjusting its weights using a training algorithm. The training algorithm adapts the weights by attempting to minimize the sum of the squared error between a desired output Do and the actual output of the network Yo given by; [12-14]

$$
E=\frac{1}{2} \sum_{o=1}^{O}\left(D_{o}-Y_{o}\right)^{2}
$$

Where, $\mathrm{O}$ is the number of neurons in the output layer. Each weight in the neural network is adjusted by adding an increment to reduce $\mathrm{E}$ as rapidly as possible. The adjustment is carried out over several training iterations until a satisfactorily small value of $\mathrm{E}$ is obtained, then this feature stored in the database. The error back-propagation algorithm has been used for this task.

In testing phase the distillation column signal passes through segmentation stage to divide it into frames as previously mentioned, then HOS estimated from these frames using different estimation methods, then discriminating feature is extracted from these estimates using MFCC and polynomial coefficient, then feature matching stage which is a matching process between the features of the new signal and the features saved in the database, and The last phase in the recognition system is the decision making. The decisionmaking module (maximum selector) takes the matching score as its input, and makes the final decision about the distillation column operation behavior.

\section{EXPERIMENTAL WORK}

This proposed approach for distillation column malfunction identification is applied on both real signals and simulated signals generated using software approved by International Atomic Energy Authority (IAEA) through the AFRA projects $\mathrm{RAF} / 8 / 040$ and $\mathrm{RAF} / 1 / 004$. Our data base contains all common malfunctions in distillation column, where it contains 105 different distillation column scanning signal, 18 signal of them represents the normal behavior of the distillation column, 18 signal represent collapsed tray malfunction, 20 signal represent foaming type malfunction, 20 signal represent weeping type malfunction, 20 signal represent damage type malfunction, and 9 signal represent flooded tray malfunction. The malfunction in all not normal data base signals may be only in one tray or more than one tray of the distillation column.

In the training phase of our approach, the training database is first constructed from 54 signal contain all types of the above mentioned distillation column malfunctions. These signals are segmented into frames; each frame contains only the signal of one tray, then HOS are estimated from these frames. The MFCCs and polynomial coefficients are estimated to form the discriminating feature matrix. The features used in all experiments are 13 MFCCs and 26 polynomial coefficients forming feature vectors of 39 coefficients for each signal. Three tests for extracting these features are adopted in the paper. In the first test, the features are extracted from the indirect estimation of the bispectrum of these signals. In the second test, the features are extracted from the bispectrum of these signals estimated by using ARMA method. In the last test, the features are extracted from the trispectrum of these signals using ARMA method.

In the testing phase, the performance of the proposed approach tested by using all the data base signals, which firstly are segmented into frames contain only tray signal, and then degraded with different types of noise to test the performance of the proposed approach in the presence of noise. The degradations considered in this approach are Gaussian and Rayleigh noise. The recognition rate is used as the performance evaluation metric in all experiments, which defined as the ratio of the number of success identifications to the total number of identification trials.

Recognition rate $=\frac{\text { Number of success identifications }}{\text { toal number of identifications }}$

\section{RESULTS AND DISCUSSION}

The performance of any practical approach appears when applying it on a noisy environment especially in our approach, because gamma scanning of a distillation column is performed in the presence of different types of noise. The proposed approach is a classification approach which means that not only it determine whether the signals are malfunction or normal, but also determine the type of that malfunction if exist and position of the malfunction tray from the whole column.

Figure 9 shows the recognition rate versus signal to noise ratio (SNR) for distillation column signals contaminated with Gaussian noise. It is clear from the figure that, the features extracted from the bispectrum of the column signals estimated using indirect method give the best results at low and high SNR. 
The question which arises from the above figure is why recognition rate approximately does not affected by SNR. This insensitivity to Gaussian noise is because HOS suppress additive colored Gaussian noise of unknown power spectrum as illustrated bove. It is not clear from the figure above which of the different estimates is useful for determining a specific type of malfunction, so it is useful to translate this figure into tables of each estimate, each table should show the error of detecting each type of distillation column malfunction and the recognition rate as illustrated in table 1 .

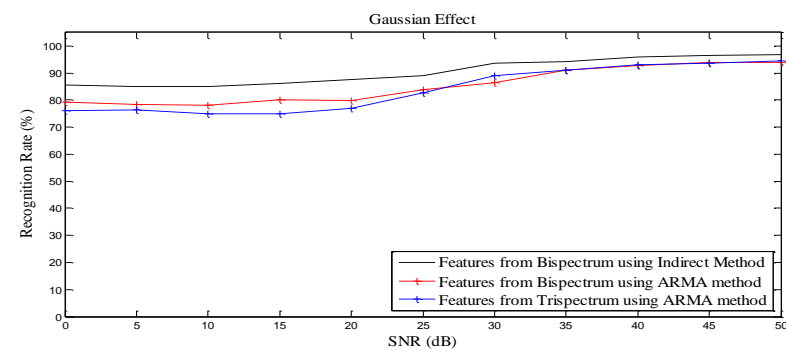

Fig 9: Distillation column malfunction identification results using the degraded signal by Gaussian noise

In order to detect the foamed trays in a distillation column, non-parametric estimator for HOS is very suitable to achieve this purpose with smaller error than other estimators; also indirect non-parametric estimator is suitable for detecting weep trays, and also it suitable for detecting collapsed trays with nearly the same percentage of error. All types of estimators can be used for detecting damaged and normal trays in a column as illustrated in table 1.

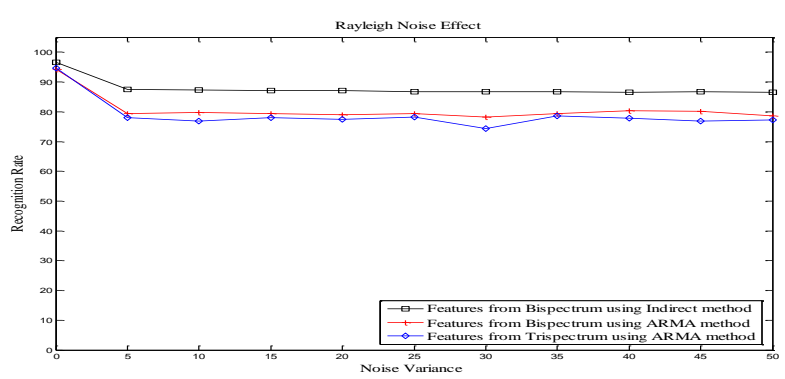

Fig 10: Distillation column malfunction identification results using the degraded signal by Rayleigh noise

Fig. 10 shows the recognition rate versus noise variance for distillation column signals contaminated with Rayleigh noise. It is clear from the figure that, the features extracted from the Bispectrum of the column signals estimated using indirect can be used efficiently for the detection and classification of all types of distillation column malfunctions. Also it is clear from the figure that all estimators can be used for this purpose but with slightly larger errors.

Table 1. Percentage of faulty detected malfunction type for different HOS estimate methods

\begin{tabular}{|c|c|c|c|c|c|c|c|}
\hline $\begin{array}{c}\text { Kaulty detected } \\
\text { signat type }\end{array}$ & $\begin{array}{c}\text { Foam } \\
\text { tray error } \\
\mathbf{\%}\end{array}$ & $\begin{array}{c}\text { Weep } \\
\text { tray error } \\
\mathbf{\%}\end{array}$ & $\begin{array}{c}\text { Damage } \\
\text { tray error } \\
\mathbf{\%}\end{array}$ & $\begin{array}{c}\text { Collapse } \\
\text { tray error } \\
\mathbf{\%}\end{array}$ & $\begin{array}{c}\text { Flooded } \\
\text { tray error } \\
\mathbf{\%}\end{array}$ & $\begin{array}{c}\text { Normal } \\
\text { tray error } \\
\text { \% }\end{array}$ & $\begin{array}{c}\text { Recognition } \\
\text { rate }\end{array}$ \\
\hline $0 \mathrm{~dB}$ & 1.2698 & 2.3280 & 2.9630 & 2.8571 & 0.4233 & 4.5503 & 85.6 \\
\hline $5 \mathrm{~dB}$ & 1.1640 & 2.3280 & 2.7513 & 2.8571 & 0.4233 & 5.3968 & 85.08 \\
\hline $10 \mathrm{~dB}$ & 1.1640 & 2.1164 & 2.4339 & 2.4339 & 0.4233 & 6.5608 & 84.8677 \\
\hline $15 \mathrm{~dB}$ & 1.3757 & 1.5873 & 1.3757 & 1.2698 & 0.4233 & 7.7249 & 86.24 \\
\hline $20 \mathrm{~dB}$ & 1.1640 & 1.6931 & 1.0582 & 0.8466 & 0.4233 & 7.0899 & 87.725 \\
\hline $25 \mathrm{~dB}$ & 1.1640 & 1.6931 & 0.8466 & 0.4233 & 0.4233 & 6.3492 & 89.1 \\
\hline $30 \mathrm{~dB}$ & 1.0582 & 1.2698 & 0.9524 & 0.2116 & 0.4233 & 4.5503 & 93.7566 \\
\hline $35 \mathrm{~dB}$ & 0.9524 & 1.1640 & 0.8466 & 0.2116 & 0.4233 & 2.1164 & 94.2857 \\
\hline $40 \mathrm{~dB}$ & 0.9524 & 0.3175 & 0.8466 & 0.1058 & 0.4233 & 1.2698 & 96.08 \\
\hline $45 \mathrm{~dB}$ & 0.8466 & 0.2116 & 0.4233 & 0.2116 & 0.4233 & 1.3757 & 96.5 \\
\hline $50 \mathrm{~dB}$ & 0.8466 & 0.1058 & 0.5291 & 0.1058 & 0.4233 & 1.2698 & 96.7 \\
\hline
\end{tabular}

(1-a) Bispectrum using indirect method

\begin{tabular}{|c|c|c|c|c|c|c|c|}
\hline $\begin{array}{c}\text { Faulty detected } \\
\text { signaltype }\end{array}$ & $\begin{array}{c}\text { Foam } \\
\text { tray error } \\
\mathbf{\%}\end{array}$ & $\begin{array}{c}\text { Weep } \\
\text { tray error } \\
\mathbf{\%}\end{array}$ & $\begin{array}{c}\text { Damage } \\
\text { tray error } \\
\mathbf{\%}\end{array}$ & $\begin{array}{c}\text { Collapse } \\
\text { tray error } \\
\mathbf{\%}\end{array}$ & $\begin{array}{c}\text { Flooded } \\
\text { tray error } \\
\mathbf{\%}\end{array}$ & $\begin{array}{c}\text { Normal } \\
\text { tray error } \\
\mathbf{\%}\end{array}$ & $\begin{array}{c}\text { Recognition } \\
\text { rate \% }\end{array}$ \\
\hline $0 \mathrm{~dB}$ & 1.5873 & 1.9048 & 1.2698 & 1.0582 & 0.9524 & 13.9683 & 79.2593 \\
\hline $5 \mathrm{~dB}$ & 1.4815 & 1.6931 & 1.7989 & 1.0582 & 0.9524 & 14.4974 & 78.5185 \\
\hline $10 \mathrm{~dB}$ & 1.3757 & 2.2222 & 1.9048 & 0.9524 & 0.9524 & 14.6032 & 77.989 \\
\hline $15 \mathrm{~dB}$ & 1.3757 & 1.6931 & 2.0106 & 1.0582 & 0.9524 & 12.8042 & 80.1052 \\
\hline $20 \mathrm{~dB}$ & 1.4815 & 1.2698 & 1.5873 & 1.0582 & 0.9524 & 13.7566 & 79.8942 \\
\hline $25 \mathrm{~dB}$ & 1.4815 & 0.8466 & 1.4815 & 1.0582 & 0.8466 & 10.3704 & 83.9153 \\
\hline $30 \mathrm{~dB}$ & 1.3757 & 0.9524 & 1.2698 & 0.9524 & 0.8466 & 8.0423 & 86.5608 \\
\hline $35 \mathrm{~dB}$ & 1.2698 & 0.8466 & 0.8466 & 0.9524 & 0.8466 & 4.1270 & 91.1111 \\
\hline $40 \mathrm{~dB}$ & 1.1640 & 0.8466 & 0.8466 & 1.0582 & 0.8466 & 2.5397 & 92.6984 \\
\hline $45 \mathrm{~dB}$ & 1.3757 & 0.4233 & 0.8466 & 0.8466 & 0.8466 & 1.5873 & 93.9683 \\
\hline $50 \mathrm{~dB}$ & 1.3757 & 0.4233 & 0.9524 & 0.8466 & 0.8466 & 1.5873 & 93.9683 \\
\hline
\end{tabular}

(1-c) Bispectrum using ARMA modeling method 


\begin{tabular}{|c|c|c|c|c|c|c|c|}
\hline $\begin{array}{c}\text { Faulty detected } \\
\text { signat type }\end{array}$ & $\begin{array}{c}\text { Foam } \\
\text { tray error } \\
\mathbf{\%}\end{array}$ & $\begin{array}{c}\text { Weep } \\
\text { tray error } \\
\mathbf{\%}\end{array}$ & $\begin{array}{c}\text { Damage } \\
\text { tray error } \\
\mathbf{\%}\end{array}$ & $\begin{array}{c}\text { Collapse } \\
\text { tray error } \\
\mathbf{\%}\end{array}$ & $\begin{array}{c}\text { Flooded } \\
\text { tray error } \\
\%\end{array}$ & $\begin{array}{c}\text { Normal } \\
\text { tray error } \\
\mathbf{\%}\end{array}$ & $\begin{array}{c}\text { Recognition } \\
\text { rate \% }\end{array}$ \\
\hline $0 \mathrm{~dB}$ & 1.3757 & 2.0106 & 2.0106 & 0.8466 & 0.6349 & 17.1429 & 75.9788 \\
\hline $5 \mathrm{~dB}$ & 1.3757 & 1.6931 & 1.9048 & 0.9524 & 0.7407 & 17.0370 & 76.2963 \\
\hline $10 \mathrm{~dB}$ & 1.3757 & 2.1164 & 1.9048 & 0.9524 & 0.7407 & 18.0952 & 74.8148 \\
\hline $15 \mathrm{~dB}$ & 1.4815 & 1.6931 & 2.0106 & 1.0582 & 0.6349 & 18.3069 & 74.8148 \\
\hline $20 \mathrm{~dB}$ & 1.2698 & 1.6931 & 1.4815 & 1.0582 & 0.7407 & 16.7196 & 77.0370 \\
\hline $25 \mathrm{~dB}$ & 1.4815 & 0.9524 & 1.3757 & 1.0582 & 0.7407 & 11.7460 & 82.6455 \\
\hline $30 \mathrm{~dB}$ & 1.2698 & 1.0582 & 1.0582 & 0.9524 & 0.7407 & 5.9259 & 88.9947 \\
\hline $35 \mathrm{~dB}$ & 1.2698 & 0.9524 & 1.1640 & 1.0582 & 0.6349 & 3.8095 & 91.1111 \\
\hline $40 \mathrm{~dB}$ & 1.3757 & 0.9524 & 0.5291 & 0.8466 & 0.8466 & 2.4339 & 93.0159 \\
\hline $45 \mathrm{~dB}$ & 1.1640 & 0.7407 & 0.8466 & 0.8466 & 0.8466 & 1.7989 & 93.7566 \\
\hline $50 \mathrm{~dB}$ & 1.0582 & 0.5291 & 0.5291 & 1.0582 & 0.7407 & 1.4815 & 94.6032 \\
\hline
\end{tabular}

(1-d) Trispectrum using ARMA modeling method

In general, the results show that HOS can be used efficiently for the distillation column malfunction identification especially when the gamma scanning of that column is performed at high noisy scanning conditions.

\section{CONCLUSION}

This paper presents an approach for the identification of distillation column malfunction depending on the estimation of higher order spectra for the distillation column signals which obtained by gamma ray scanning of that column, then discriminating features have been extracted from these signals using MFCC and polynomial coefficient. These discriminating features have been used for training a neural network to classify the obtained signals to the different types of distillation column malfunction. The results show that HOS can be used efficiently for the distillation column malfunction identification especially at high noisy scanning conditions.

\section{ACKNOWLEDGMENT}

The practical work is supported by IAEA through the project number RAF/1/004. So, it gives me the greatest pleasure to thank Dr. Horya A. M. Arafa from Engineering Department, Nuclear Research Center, Atomic Energy Authority for her valuable discussions and support.

\section{REFERENCES}

[1] V. A. S. Pablo, F. E. Costa, P. R. Rela, W. A. P. Calvo, and M. M. Hamada, "Gamma Scanning Evaluation for Random Packed Columns", IEEE Nuclear Science Symposium Conference, pp. 519-523, 2005.

[2] K. Salahshoor and S. GHaribshaiyan, "Online Multivariable Identification of a Nonlinear Distillation Column using an Adaptive Takagi-Sugeno Fuzzy Model”, IEEE Conference, pp. 527-532, 2008.

[3] M. Muddu and C. S. Patwardhan, "Adaptive Predictive Control of a High Purity Distillation Column Using Irregularly Sampled Multi-rate Data", International Symposium, pp. 192-197, 2011.

[4] D. Ugryumova, G. Vandersteen, B. Huyck, F. Logist, J. V. Impe, and B. De Moor, "Identification and Modeling of Distillation Columns From Transient Response Data", Instrumentation and Measurement Technology Conference (I2MTC), IEEE conference, pp. 2098-2103, 2012.
[5] M. Sanches, M. Hanaguchi, F. Beckmann and W. Calvo, "Radiological Safety in the Gamma Scan Procedures", Proc. of 2007 International Nuclear Atlantic Conference, Santos, Brazil, 2007.

[6] P. Vasquez, C. Mesquita, G. LeRoux and M. Hamada, "Methodological Analysis of Gamma Tomography System for Large Random Packed Columns", 7th International Topical Meeting on Industrial Radiation and Radioisotope Measurements Application, Prague, Czech Republic, 2008.

[7] C. A. P. Wilson, H. M. Margarida, F. E. Sprenger, P. A. S. Vasquez, P. R. Rela, J. F. T. Martins, J. C. S. Pereira, N. M. Omi and C. H. Mesquita, "Gamma-ray Computed Tomography Scanners for Applications in Multiphase System Columns", Nukleonika, Vol. 54, No. 2, pp.129-133, 2009.

[8] Dr Jaafar, "Gamma-Ray Scanning for Troubleshooting, Optimization and Predictive Maintenance of Distillation Columns", Hydrocarbon Asia, pp. 62-65, 2005.

[9] A.E Hills, "A window into Radioactive Sealed Source Diagnostics", IAEA/AFRA Regional Training Course on Capacity Building for Enhanced Gamma Scanning of Industrial Process, Tunis, Tunisia, October 2012.

[10] C. L. Nikias and J. M. Mendel, "Signal Processing with Higher Order Spectra", IEEE Signal Processing Magazine, pp. 10-37, July 1993.

[11] J. G. Proakis, C. M. Rader, F. Ling, and C. L. Nikias, "Advanced Digital Signal Processing", Macmillan Publishing Company, a division of Macmillan Inc. 1992.

[12] R.V Pawar, P.P. Kajave and S .N. Mali, " Speaker Identification using Neural Networks", Proceedings of World Academy of Science, Engineering and Technology, Vol. 7, pp. 1307- 6884, 2005.

[13] G. Dreyfus, "Neural Networks Methodology and Applications," Springer Verlag Berlin Heidelberg, 2005.

[14] A. I. Galushkin, "Neural Networks Theory," SpringerVerlag Berlin Heidelberg, 2007. 